\title{
Angiotensin-(1-7) attenuates organ injury and mortality in rats with polymicrobial sepsis
}

Hsin-Jung Tsai ${ }^{1+}$, Mei-Hui Liao ${ }^{2 \dagger}$, Chih-Chin Shih², Shuk-Man Ka ${ }^{3}$, Cheng-Ming Tsao ${ }^{1,4^{*}}$ (D) and Chin-Chen Wu ${ }^{2}$

\begin{abstract}
Background: Sepsis and related multiple organ dysfunction result in high morbidity and mortality. Angiotensin (Ang)-(1-7), a biologically active peptide, has various opposing effects of Ang II. Because the effect of Ang-(1-7) on sepsis is unknown, in this study we aimed to determine the impact of Ang-(1-7) on pathophysiologic changes in a clinically relevant model of polymicrobial sepsis induced by cecal ligation and puncture (CLP).

Methods: Sepsis was induced by CLP in rats under anesthesia. Rats were randomized to one of the following five groups: (1) sham-operated group, (2) Ang-(1-7) (1 mg/kg intravenously infused for $1 \mathrm{~h})$ at $3 \mathrm{~h}$ and $6 \mathrm{~h}$ after sham operation, (3) CLP, (4) Ang-(1-7) at $3 \mathrm{~h}$ after CLP, and (5) Ang-(1-7) at $3 \mathrm{~h}$ and $6 \mathrm{~h}$ after CLP. Rats were observed for $24 \mathrm{~h}$ after CLP surgery and then killed for subsequent histological examination.

Results: Ang-(1-7) significantly improved the survival of septic rats $(83.3 \%$ vs. $36.4 \%$ at $24 \mathrm{~h}$ following CLP; $p=0$. 009). Ang-(1-7) attenuated the CLP-induced decreased arterial pressure and organ dysfunction, indicated by diminished biochemical variables and fewer histological changes. Ang-(1-7) significantly reduced the level of plasma interleukin-6 and pulmonary superoxide production $(p<0.05)$. Moreover, caspase-3 and cytoplasmic IKB expression in liver was significantly lower in the Ang-(1-7)-treated CLP rats $(p<0.05)$.

Conclusions: In this clinically relevant model of sepsis, Ang-(1-7) ameliorates CLP-induced organ dysfunction and improves survival, possibly through suppressing the inflammatory response, oxidative stress, and apoptosis, suggesting that Ang-(1-7) could be a potential novel therapeutic approach to treatment of peritonitis and polymicrobial sepsis.
\end{abstract}

Keywords: Angiotensin-(1-7), Apoptosis, Inflammatory response, Organ injury, Polymicrobial sepsis

\section{Background}

Sepsis remains the major cause of mortality and morbidity in intensive care $[1,2]$, despite significant advances in understanding of the biologic alterations and improvements in medical care. Sepsis is caused by the release of proinflammatory cytokines and ROS, as well as tissue cell apoptosis, leading to development of multiple organ injuries [3]. In addition, cross-talk between these

\footnotetext{
*Correspondence: cm.cmtsao@gmail.com

${ }^{+}$Hsin-Jung Tsai and Mei-Hui Liao contributed equally to this work.

'Department of Anesthesiology, Taipei Veterans General Hospital and National Yang-Ming University, No. 201, Sec. 2, Shipai Road, Beitou District, Taipei 11217, Taiwan, Republic of China

${ }^{4}$ Department of Anesthesiology, Tri-Service General Hospital, National

Defense Medical Center, Taipei, Taiwan, Republic of China

Full list of author information is available at the end of the article
}

processes has been recognized in sepsis [3]. However, no specific and effective pharmacological intervention for sepsis is currently available.

Angiotensin (Ang)-(1-7), a biologically active peptide of the renin-angiotensin system (RAS), is produced mainly through angiotensin-converting enzyme 2 (ACE2) using primarily angiotensin II (Ang II) as a substrate $[4,5]$. Through binding its $\mathrm{G}$ protein-coupled receptor Mas [6], Ang-(1-7) counteracts various effects of Ang II, such as vasoconstriction, inflammation, proliferation, and apoptosis [7]. Therefore, the ACE2/Ang-(1-7)/ Mas axis contributes to the regulation of vascular tone, as well as inflammatory responses and organ function after injury $[5,8]$.

(c) The Author(s). 2018 Open Access This article is distributed under the terms of the Creative Commons Attribution 4.0 International License (http://creativecommons.org/licenses/by/4.0/), which permits unrestricted use, distribution, and reproduction in any medium, provided you give appropriate credit to the original author(s) and the source, provide a link to the Creative Commons license, and indicate if changes were made. The Creative Commons Public Domain Dedication waiver (http://creativecommons.org/publicdomain/zero/1.0/) applies to the data made available in this article, unless otherwise stated. 
Accumulating evidence indicates that ACE2 plays an important role in severe lung injury $[9,10]$, and it attenuates endotoxin-induced lung via the Ang-(1-7)/Mas pathway by inhibiting NF- $\mathrm{kB}$ activation [11]. It has been reported that Ang-(1-7) has its anti-inflammatory effects in arthritis and allergic airway through the Mas receptor [12, 13] and decreases lipopolysaccharide (LPS)-stimulated proinflammatory cytokines, including tumor necrosis factor- $\alpha$ and interleukin 6 (IL-6), in mouse peritoneal macrophages [14]. Moreover, Ang-(17) attenuates cytokine production, hypothermia, and mortality from LPS-induced endotoxemia; however, the underlying molecular mechanism remains elusive [15]. In addition, apoptosis plays a major role in the pathogenesis of sepsis [16], which is involved in sepsis-induced organ dysfunction [3]. Furthermore, Ang-(1-7)/Mas signaling could inhibit LPS-induced apoptosis in alveolar epithelial cells [17] and pulmonary microvascular endothelial cells [18].

A variety of animal species have been used to study septic responses induced by endotoxin. However, cecal ligation and puncture (CLP) is the most frequently used model of polymicrobial sepsis because it closely resembles the progression and characteristics of human sepsis [19]. Therefore, we applied a CLP approach to induce sepsis in rats in this study, and we investigated whether Ang-(1-7) exerts beneficial effects against CLP-induced organ injuries and death via suppressing inflammation and apoptosis pathways.

\section{Methods}

We used male adult Wistar rats (280 to 320 g; BioLASCO Taiwan Co., Taipei, Taiwan) in this study. The animals were housed in 12-h/12-h light/dark conditions with free access to food and tap water. This study was approved by the local institutional review board, and the experiments were performed in compliance with the $\mathrm{Na}$ tional Institutes of Health guidelines for the treatment of animals and for ethical animal research.

\section{Surgical procedures}

Under anesthesia with intraperitoneal sodium pentobarbital $(50 \mathrm{mg} / \mathrm{kg})$, polyurethane catheters were inserted into the left carotid artery for blood pressure monitoring and blood sampling and into the right internal jugular vein for drug administration. Adequate anesthesia in animals was verified by pinching their toes. The catheters were exteriorized and fixed to the back of the neck. Subsequently, the cannulated rats were allowed to recover to their normal condition with standardized pellet food and tap water ad libitum.

On the next day, intraperitoneal sepsis was induced by CLP under anesthesia with $50 \mathrm{mg} / \mathrm{kg}$ intravenous pentobarbital [20]. Briefly, a laparotomy was made through a 2-cm-long midline incision in the lower abdomen. The cecum was exposed and ligated with a 3-0 silk ligature just distal to the ileocecal valve, punctured twice at opposite ends using an 18-gauge needle. The bowel was then returned into the abdominal cavity, and the incision was closed. In addition, $0.2 \%$ lidocaine was used to infiltrate the incision wound after abdominal closure for analgesia. The rats in the sham operation (SOP) group underwent similar laparotomy and cecal exposure without CLP. All animals were treated subcutaneously with $0.9 \% \mathrm{NaCl}$ solution $(10 \mathrm{ml} / \mathrm{kg})$ immediately after laparotomy.

All rats enrolled in the study were kept in a small in-house animal facility to enable optimal monitoring, and their overall health status was checked every $3 \mathrm{~h}$ for signs of distress, such as shallow and rapid respiration, piloerection, and hunching or cowering in the corner of the cage. Rats were killed only $24 \mathrm{~h}$ following CLP or sham surgery or upon signs of imminent death (that is, unresponsive to external stimuli, inability to maintain an upright position, tremor, and prolonged/deep hypothermia and/or agonal breathing) via an overdose of pentobarbital $(100 \mathrm{mg} / \mathrm{kg}$ intravenously). The survival rate during the study in each group was further analyzed. In regard to "blinding" implemented in this study, an investigator blinded to the treatment group made the assessment for survival. In addition, analysis of the pathologic examination and the statistical results were done in a blinded fashion.

\section{Experimental protocol}

Rats received CLP or SOP in randomized fashion by flipping a coin to one of five treatment groups (Fig. 1): (1) SOP group: an intravenous infusion of $0.9 \% \mathrm{NaCl}$ for $1 \mathrm{~h}$ at 3 and $6 \mathrm{~h}$ after SOP $(n=6)$, (2) SOP-Ad group: an intravenous infusion of $1 \mathrm{mg} / \mathrm{kg}$ Ang-(1-7) for $1 \mathrm{~h}$ at 3 and $6 \mathrm{~h}$ after SOP $(n=6)$, (3) CLP group: same regimen and volume of saline at 3 and $6 \mathrm{~h}$ after CLP procedure $(n=8)$, (4) CLP-As group: same regimen of Ang-(1-7) at $3 \mathrm{~h}$ after CLP procedure $(n=3)$, or (5) CLP-Ad group: same regimen of Ang-(1-7) at 3 and $6 \mathrm{~h}$ after CLP procedure $(n=8)$. Ang-(1-7) (Tocris Bioscience, Bristol, $\mathrm{UK}$ ) was dissolved in $0.9 \% \mathrm{NaCl}$, and its dosage was selected according to a previous study [15].

\section{Measurement of hemodynamic parameters}

At 0,3 and 24 h after CLP or SOP in all animals, mean arterial pressure (MAP) and heart rate (HR) were monitored by connecting the arterial catheter to a pressure transducer (P23ID; Statham, Oxnard, CA, USA) and displaced on a polygraph recorder (MacLab/4e; AD Instruments Pty Ltd., Castle Hill, Australia).

\section{Quantification of organ function}

At 0,3 and $24 \mathrm{~h}$ following CLP or sham surgery, $1 \mathrm{ml}$ of arterial blood was collected, and $10 \mu \mathrm{l}$ of the blood 


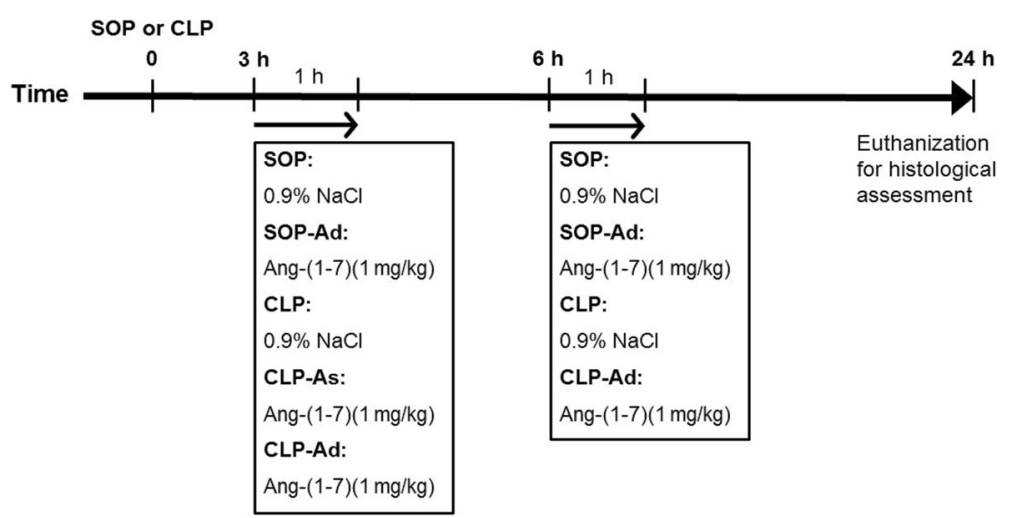

Fig. 1 Experimental design. Timeline of 24-h experiments. SOP = sham operation, $0.9 \% \mathrm{NaCl}=$ normal saline (intravenously for $1 \mathrm{~h}$ ), Ang-(1-7)= angiotensin-(1-7) (1 mg/kg intravenously for $1 \mathrm{~h})$, SOP-Ad =Ang-(1-7) at 3 and $6 \mathrm{~h}$ after sham operation, CLP-Ad=Ang-(1-7) at 3 and $6 \mathrm{~h}$ after $\mathrm{CLP}$ procedure, $\mathrm{CLP}-\mathrm{As}=\mathrm{Ang}-(1-7)$ at $3 \mathrm{~h}$ only after CLP procedure

sample was analyzed for blood glucose by using a One-Touch II blood glucose monitoring system (Lifescan Inc., Milpitas, CA, USA). The remainder of the collected blood was then centrifuged ( $3 \mathrm{~min}, 16,000 \mathrm{~g}$ ) and analyzed for plasma levels of alanine aminotransferase (ALT), blood urea nitrogen (BUN), creatinine, and lactate dehydrogenase (LDH) by Fuji DRICHEM 3030 (Fuji Photo Film Co., Ltd., Tokyo, Japan). Each volume of blood withdrawn was replaced immediately by the infusion of an equal volume of saline. Owing to blood clots or kinking of the arterial catheter, blood samples could not be obtained in some of the rats surviving the whole experimental period, especially in rats that underwent the CLP procedure.

\section{Measurement of plasma IL-6 concentrations}

Collected plasma at 0 and $24 \mathrm{~h}$ after sham or CLP surgery was analyzed for IL-6 in duplicate by using enzyme-linked immunosorbent assay kits (R\&D Systems, Inc., Minneapolis, MN, USA) according to the manufacturer's instructions.

\section{Measurement of superoxide production}

At the end of the experiment, the tissues of lung, liver, and kidney were removed and placed on the scintillation plates. These microplates containing Krebs-HEPES buffer $(100 \mu \mathrm{l})$ and $1.25 \mathrm{mM}$ lucigenin $(50 \mu \mathrm{l})$ were placed into a microplate luminometer (Hidex Microplate Luminometer, Turku, Finland) for analysis of superoxide ion as previously described [20]. The obtained values were measured in duplicate, and all tissues were then dried in a laboratory oven for $24 \mathrm{~h}$. The superoxide levels in all tissues were expressed as counts per second in each milligram of dry tissue.

\section{Western blot analysis}

For cleaved caspase-3 and nuclear factor of kappa light polypeptide gene enhancer in B-cells inhibitor ( $\mathrm{I} \mathrm{B}$ ) expression analysis $[11,18,21]$, supernatants of homogenized liver tissues $(100 \mu \mathrm{g}$ total protein) were separated by electrophoresis in a $10-12 \%$ polyacrylamide gel and transferred onto nitrocellulose membrane (Mini Trans-Blot Cell; Bio-Rad Laboratories, Hercules, CA, USA). The membranes were blocked with 5\% albumin (BioShop Canada Inc., Burlington, ON, USA) in Tris-buffered solution containing $0.1 \%$ Tween-20 (TBST) for $1.5 \mathrm{~h}$ at room temperature and then incubated overnight at $4{ }^{\circ} \mathrm{C}$ with primary antibody (cleaved caspase 3, 1:500 dilution, Cell Signaling Technology Inc., Danvers, MA, USA; IкB, 1:5000 dilution, Abcam, Cambridge, UK) in TBST buffer, which was followed by the addition of a horseradish peroxidase-conjugated goat antirabbit immunoglobulin G (Cell Signaling Technology Inc.). The protein expression was visualized using Pierce enhanced chemiluminescence Western blotting reagent (Thermo Fisher Scientific, Rockford, IL, USA), followed by exposure to radiographic film. The densitometry of bands was quantified.

\section{Histological examination}

Specimens of liver and lung were fixed in $10 \%$ formaldehyde for more than $24 \mathrm{~h}$, which was followed by dehydration in graded ethanol. The tissues were embedded in paraffin wax, sectioned into 4- $\mu$ m-thick slices, and stained with hematoxylin and eosin. The severity of lung injury (lung edema, pulmonary congestion, thickening of the alveolar wall, and areas of inflammatory infiltration) and liver necrosis was scored from 0 (none or minimal) to 3 (severe) by a pathologist in a blinded fashion in three animals from each group. The score of ten randomly selected high-power fields was used for assessment.

\section{Statistical analysis}

Shapiro-Wilk tests were used to evaluate whether collected variables were normally distributed. Variables that 
did not follow the normal distribution were $\log$-transformed. Analysis of variance for repeated measures was used to compare physiological parameters among groups. For the remaining parameters (including superoxide production and caspase- 3 and IкB expression), the significance of differences was analyzed by one-way analysis of variance combined with the Newman-Keuls post hoc test. The severity score of tissue injury was compared between the CLP and CLP-Ad groups by the Mann-Whitney $U$ test. Survival distribution was compared using the log-rank test and represented by Kaplan-Meier curves. Data are presented as median and IQR. A $p$ value less than 0.05 was considered statistically significant. Regarding the power analysis of our findings, Schoenfeld's formula for survival models was used to estimate post hoc power. Given a two-sided type I error rate of 0.05 , a hazard ratio of 2 , and our sample size of 12 and 22 in the CLP-Ad and CLP groups, respectively, a power level of more than 0.95 could be attained in the corresponding analyses.

\section{Results}

\section{Survival}

Comparing the overall survival, there was a significant difference among the five study groups $(p=0.002)$. In the CLP group, 2 of 22 animals died at $9 \mathrm{~h}$ (survival: 20 of 22 rats, $90.9 \%$ ), and 14 of 22 rats died at $24 \mathrm{~h}$ after CLP procedure (survival: 8 of 22 rats, 36.4\%), whereas no mortality was observed within $24 \mathrm{~h}$ in all SOP groups (survival: 6 of 6 rats, 100\% in each SOP group) (Fig. 2). In the CLP-As group, one of six rats died at $9 \mathrm{~h}$ (survival: five of six rats, $83.3 \%$ ), and three of six rats died at $24 \mathrm{~h}$ after CLP procedure (survival: three of six rats, $50 \%)$. In the CLP-Ad group, however, no mortality was observed at 9 h (survival: 12 of 12 rats, 100\%), and only 2 of 12 rats died at $24 \mathrm{~h}$ after CLP procedure (survival: 10 of 12 rats, 83.3\%). Thus, survival was higher in CLP rats with double-dose Ang-(1-7) treatment $(p=0.009$ vs. CLP group).

\section{Cardiovascular changes}

As shown in Fig. 3, baseline data of MAP and HR did not significantly differ among groups. After the CLP procedure, MAP was significantly decreased at $24 \mathrm{~h}(p<$ 0.001 vs. SOP group) (Fig. 3a). This CLP-induced severe hypotension was significantly attenuated by double-dose Ang-(1-7) ( $p=0.037$ vs. CLP group) (Fig. 3a), but not by single-dose Ang-(1-7). Meanwhile, CLP led to a significant and sustained increase in HR from 3 to $24 \mathrm{~h}(p<$ 0.01 vs. SOP group) (Fig. $3 b)$, which was not attenuated in rats treated with Ang-(1-7) ( $p=0.42$ vs. CLP group) (Fig. 3b). In addition, MAP and HR in Ang-(1-7)-treated SOP rats did not significantly differ from the SOP rats treated with saline during the experimental period.
Plasma indexes of organ injury and blood glucose We log-transformed for the biochemical markers, including LDH, ALT, BUN, and creatinine, to normalize these data and for statistical analysis. Baseline biochemical data did not significantly differ among all groups (Fig. 4). In the SOP and SOP-Ad groups, there were no significant changes observed in the biochemical parameters during the experimental period. However, CLP procedure resulted in significant increases in the plasma levels of LDH, ALT, BUN, and creatinine at $24 \mathrm{~h}(p<$ 0.01 vs. SOP group) (Fig. $4 a-d)$. These increased parameters of organ injury were significantly ameliorated by double-dose Ang-(1-7) $\quad(p<0.01$ vs. CLP group) (Fig. 4a-d), although no significant improvement was found after the administration of single-dose Ang-(1-7).

Following the CLP procedure, the blood glucose level in animals appeared to show a biphasic change: an early elevation at $3 \mathrm{~h}$ after CLP and then a late decrease at $24 \mathrm{~h}$ after CLP ( $p<0.001$ vs. SOP group) (Fig. 4e). However, the late hypoglycemia was significantly ameliorated by double-dose Ang-(1-7) ( $p=0.018$ vs. CLP group) (Fig. 4e), although no significant improvement was found after the administration of single-dose Ang-(1-7).

\section{Plasma IL-6 level and tissue superoxide level}

After log transformation, the data of IL-6 and superoxide were normalized and used for statistical analysis. Baseline plasma level of IL-6 did not significantly differ among all groups (Fig. 5a). There were no significant changes observed in the plasma IL- 6 level in the SOP and SOP-Ad groups at the end of this study, suggesting neither the sham procedure nor Ang-(1-7) administration could change the plasma level of IL-6. However, CLP led to a significant increase in the plasma level of IL-6 at $24 \mathrm{~h}$ ( $p<0.001$ vs. SOP group) (Fig. 5a), which was significantly attenuated by double-dose Ang-(1-7) ( $p=0.002$ vs. CLP group) (Fig. 5a).

A significant increase of superoxide production was observed in lung ( $p=0.048)$ but not in liver and kidney tissue homogenates in CLP rats when compared with control rats (Fig. 5b). Ang-(1-7) significantly attenuated the increase of superoxide levels in lung tissues in CLP rats ( $p=0.039$ vs. CLP group) (Fig. $5 b$ ).

In the CLP-As group, neither the hemodynamic parameters nor the organ injury indexes showed significant improvement after treatment of CLP rats with single-dose Ang-(1-7). Therefore, oxidative stress, apoptosis, and histological studies were not further performed in this group according to the 3R (reduction, replacement, refinement) spirit.

\section{Cleaved caspase- 3 and IKB expression in liver}

To assess the effect of Ang-(1-7) on the apoptosis pathway, cleaved caspase-3 expression was determined. 


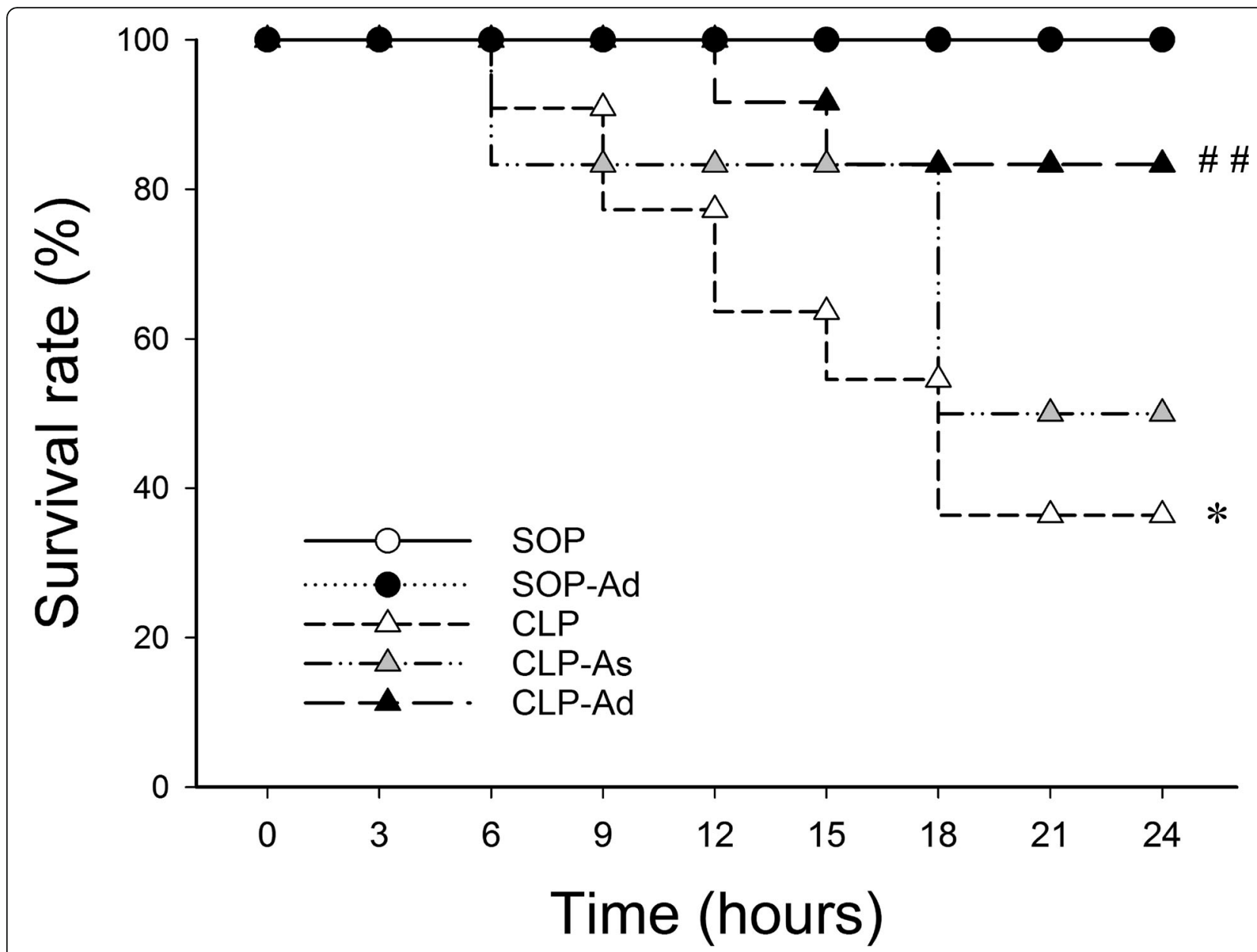

Fig. 2 Angiotensin-(1-7) improves survival after polymicrobial sepsis. Analysis of the survival rate after the administration of angiotensin-(1-7) to rats that underwent sham operation (SOP, $n=6)$, SOP plus angiotensin- $(1-7)$ administration $(1 \mathrm{mg} / \mathrm{kg}$ at 3 and $6 \mathrm{~h}$ after SOP procedure [SOP-Ad], $n=6)$, cecal ligation and puncture (CLP, $n=22), C L P$ plus angiotensin-(1-7) administration (1 $\mathrm{mg} / \mathrm{kg}$ at $3 \mathrm{~h}$ after CLP procedure [CLP-As], $n=6)$, and CLP plus angiotensin-(1-7) administration $(1 \mathrm{mg} / \mathrm{kg}$ at 3 and $6 \mathrm{~h}$ after CLP procedure [CLP-Ad], $n=12)$. Survival time was measured for $24 \mathrm{~h}$. Survival distribution was compared using the log-rank test and represented by Kaplan-Meier curves. Data are expressed as percentage of rats that survived at the end of the experiment. ${ }^{*} p<0.05$ CLP. vs. SOP. ${ }^{\# \#} p<0.01$ CLP-Ad vs. CLP

Compared with the SOP and SOP-Ad groups, CLP led to an elevated level of cleaved caspase- 3 expression in liver ( $p=0.009$ vs. SOP group), which was significantly alleviated by Ang-(1-7) administration ( $p=0.018$ vs. CLP group) (Fig. 5c). To assess the effect of Ang-(1-7) on the NF- $\kappa B$ signaling pathway, the cytoplasmic ІкB expression was determined. Compared with the SOP and SOP-Ad groups, the CLP procedure led to a decreased level of IKB in the cytoplasm ( $p=0.04$ vs. SOP group). However, treatment with Ang-(1-7) significantly inhibited the CLP-induced increase in the cytoplasmic IкB expression in liver ( $p=0.016$ vs. CLP group) (Fig. $5 \mathrm{~d}$ ).

\section{Histological studies}

The lung tissues of the CLP group showed several obvious inflammatory changes, such as lung edema, pulmonary congestion, thickening of the alveolar wall, and areas of inflammatory infiltration at $24 \mathrm{~h}$ after the CLP procedure (Fig. 6a). Treatment with Ang-(1-7) seems to alleviate the histopathological changes induced by CLP (Fig. 6a). In addition, the scores were evaluated to determine the thickness of alveolar walls and the increased number of infiltration cells, and the mean pathological score showed no significant difference in CLP rats with Ang-(1-7) treatment compared with CLP rats (1.8 [1.6 to 2] vs. 1 [ 1 to 1.4 ] , $p=0.178$ CLP vs. CLP-Ad). However, the liver tissues of CLP group showed necrotic changes, and Ang-(1-7) administration markedly alleviated the histopathological changes induced by CLP (Fig. 6b). The pathological scores of liver necrosis in CLP rats were significantly reduced by treatment of Ang-(1-7) compared with CLP rats (3 [2.5 to 3$]$ vs. 0 [0 to 0$], p=0.034$ CLP vs. CLP-Ad). 


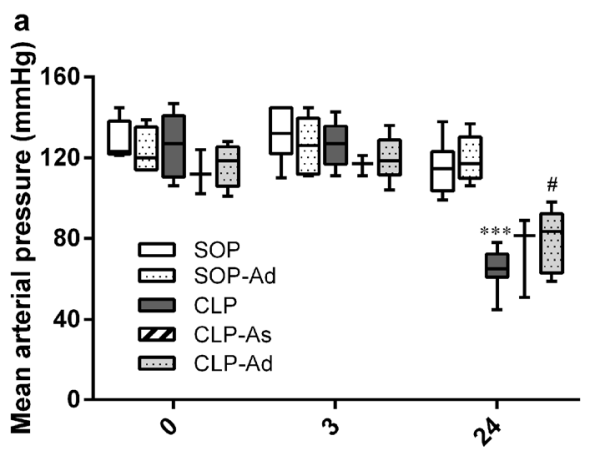

Time (hours) b

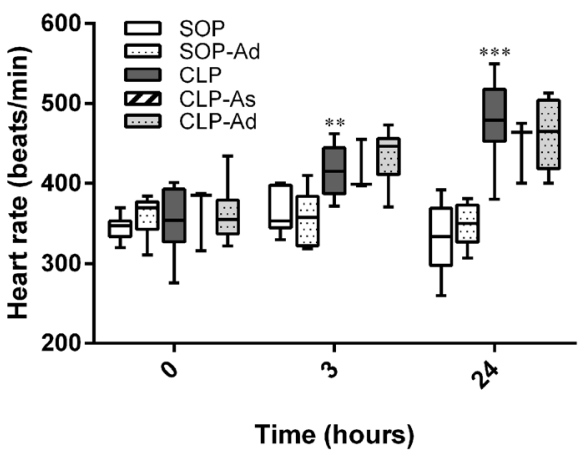

Fig. 3 Effects of angiotensin-(1-7) on changes in hemodynamic parameters in rats that underwent cecal ligation and puncture. Depicted are the changes in (a) mean arterial blood pressure and (b) heart rate during the experimental period. Rats underwent sham operation (SOP, $n=6)$, SOP plus angiotensin-(1-7) administration ( $1 \mathrm{mg} / \mathrm{kg}$ at 3 and $6 \mathrm{~h}$ after SOP procedure [SOP-Ad], $n=6)$, cecal ligation and puncture (CLP, $n=8), \mathrm{CLP}$ plus angiotensin-(1-7) administration (1 mg/kg at $3 \mathrm{~h}$ after CLP procedure [CLP-As], $n=3)$, and CLP plus angiotensin-(1-7) administration (1 mg/ $\mathrm{kg}$ at 3 and $6 \mathrm{~h}$ after CLP procedure [CLP-Ad], $n=8$ ). Analysis of variance for repeated measures was used for analysis of time $\times$ group interaction. Data are shown as median and IQR. ${ }^{* *} p<0.01,{ }^{* * *} p<0.001$ CLP. vs. SOP; ${ }^{*} p<0.05$ CLP-As or CLP-Ad vs. CLP

a

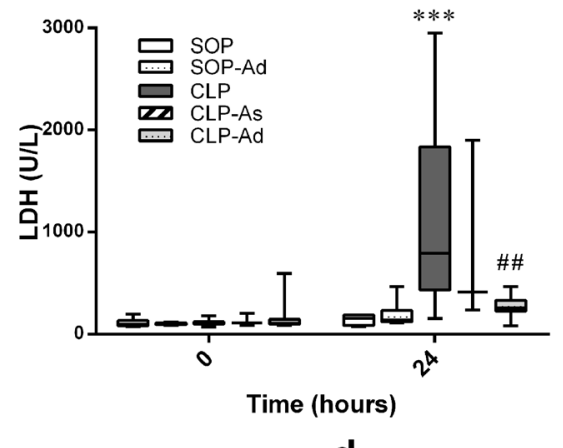

C

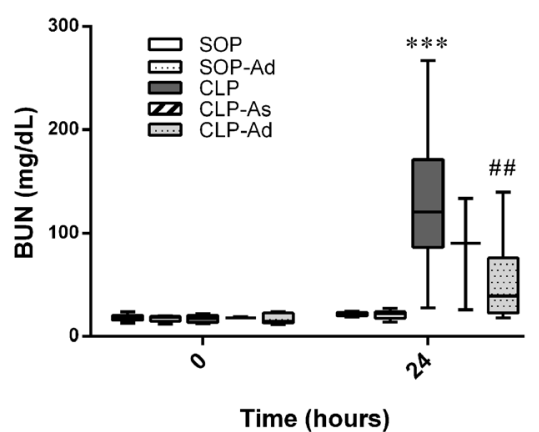

d

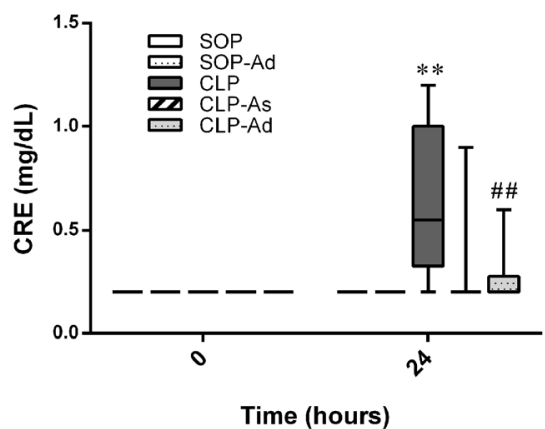

b

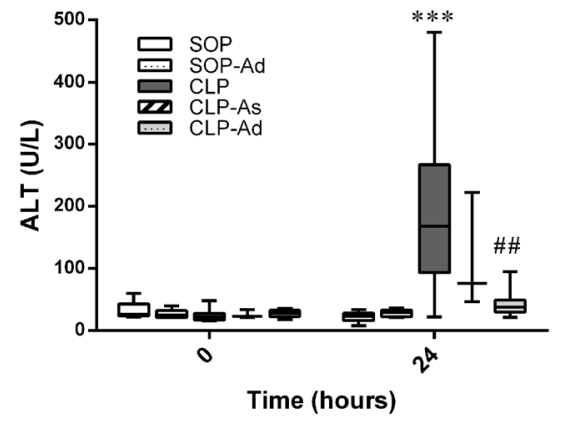

e

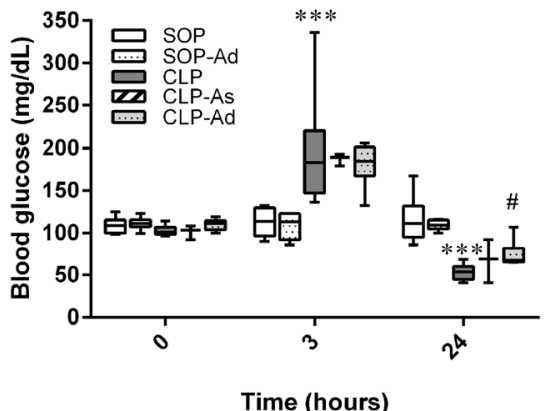

Fig. 4 Effects of angiotensin-(1-7) on biochemical parameters in rats that underwent cecal ligation and puncture. These parameters include (a) lactate dehydrogenase, (b) alanine aminotransferase (ALT), (c) blood urea nitrogen, (d) creatinine (CRE), and (e) blood glucose during the experimental period. Rats underwent sham operation (SOP, $n=6)$, SOP plus angiotensin-(1-7) administration ( $1 \mathrm{mg} / \mathrm{kg}$ at 3 and $6 \mathrm{~h}$ after SOP procedure [SOP-Ad], $n=6)$, cecal ligation and puncture (CLP, $n=8), C L P$ plus angiotensin-(1-7) administration (1 mg/kg at $3 \mathrm{~h}$ after CLP procedure [CLP-As], $n=3)$, and CLP plus angiotensin-(1-7) administration ( $1 \mathrm{mg} / \mathrm{kg}$ at 3 and $6 \mathrm{~h}$ after CLP procedure [CLP-Ad], $n=8)$. Owing to blood clots or kinking of the arterial catheter, blood samples could not be obtained in some of rats surviving the whole experimental period. Analysis of variance for repeated measures was used for analysis of time $\times$ group interaction. Data are shown as median and IQR. ${ }^{* *} p<0.01,{ }^{* * *} p$ $<0.001 \mathrm{CLP}$. vs. SOP; ${ }^{\#} p<0.05,{ }^{\# \#} p<0.01 \mathrm{CLP}+$ As or CLP + Ad vs. CLP 

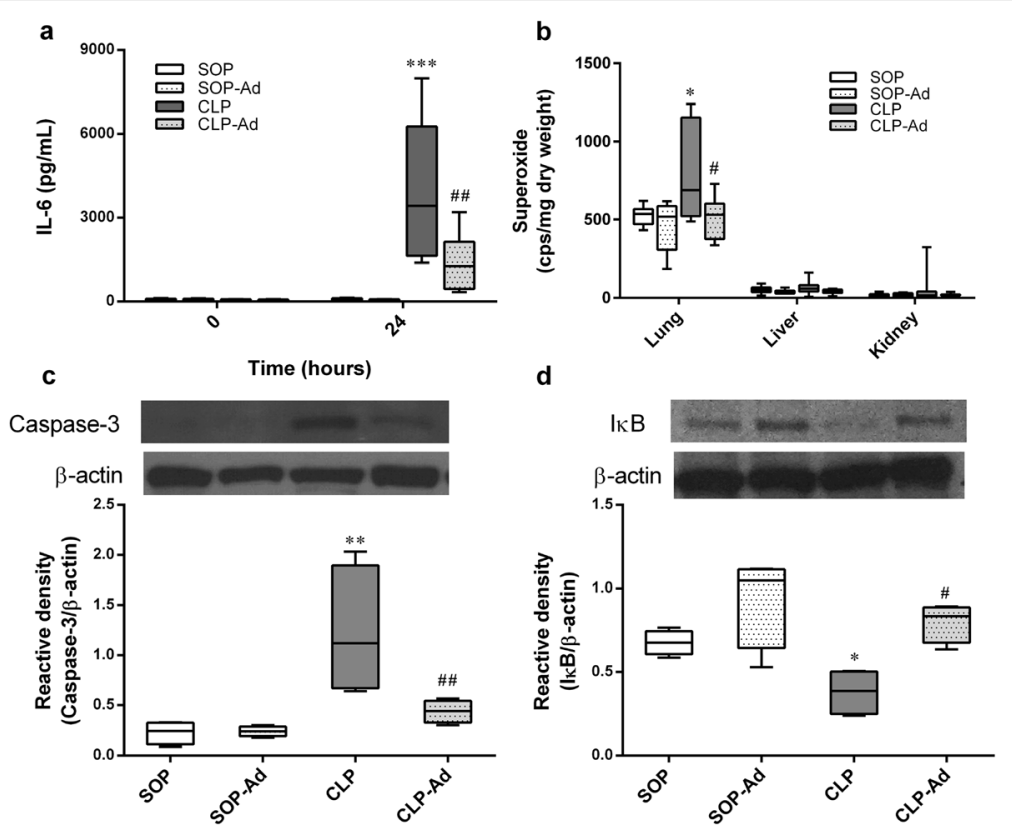

Fig. 5 Effects of angiotensin-(1-7) on plasma interleukin- 6 and tissue superoxide levels $24 \mathrm{~h}$ after procedure. Depicted are the changes in (a) plasma interleukin-6 levels and (b) tissue superoxide levels in rats that underwent sham operation (SOP, $n=5)$, SOP plus angiotensin-(1-7) administration ( $1 \mathrm{mg} / \mathrm{kg}$ at 3 and $6 \mathrm{~h}$ after SOP procedure [SOP-Ad], $n=5)$, cecal ligation and puncture (CLP, $n=8)$, and CLP plus angiotensin-(17) administration (same regimen after CLP procedure [CLP-Ad], $n=8$ ). Immunoblot analysis of (c) cleaved caspase-3 expression and (d) the cytoplasmic nuclear factor of kappa light polypeptide gene enhancer in B-cells inhibitor (IKB) in the liver. Rats underwent SOP, SOP plus angiotensin-(1-7) administration (SOP-Ad), CLP, and CLP plus angiotensin-(1-7) administration (CLP-Ad). Liver tissues were harvested at $24 \mathrm{~h}$ after surgery. Representative blots are shown on the upper panel, and $\beta$-actin served as a loading control. $n=4$ for each group. Analysis of variance (ANOVA) for repeated measures was used for interleukin-6 analysis, and one-way ANOVA combined with the Newman-Keuls post hoc test was used for the remaining parameters in between-group comparisons. The $p$ values for one-way ANOVA for superoxide production, cleaved caspase3 , and IKB expression are 0.02, 0.0035, and 0.0038, respectively. Data are shown as median and IQR. ${ }^{*} p<0.05 ;{ }^{* *} p<0.01$; ${ }^{* *} p<0.001$, CLP Vs. SOP; ${ }^{\#} p<0.05 ; \# p<0.01$, CLP-Ad vs. CLP
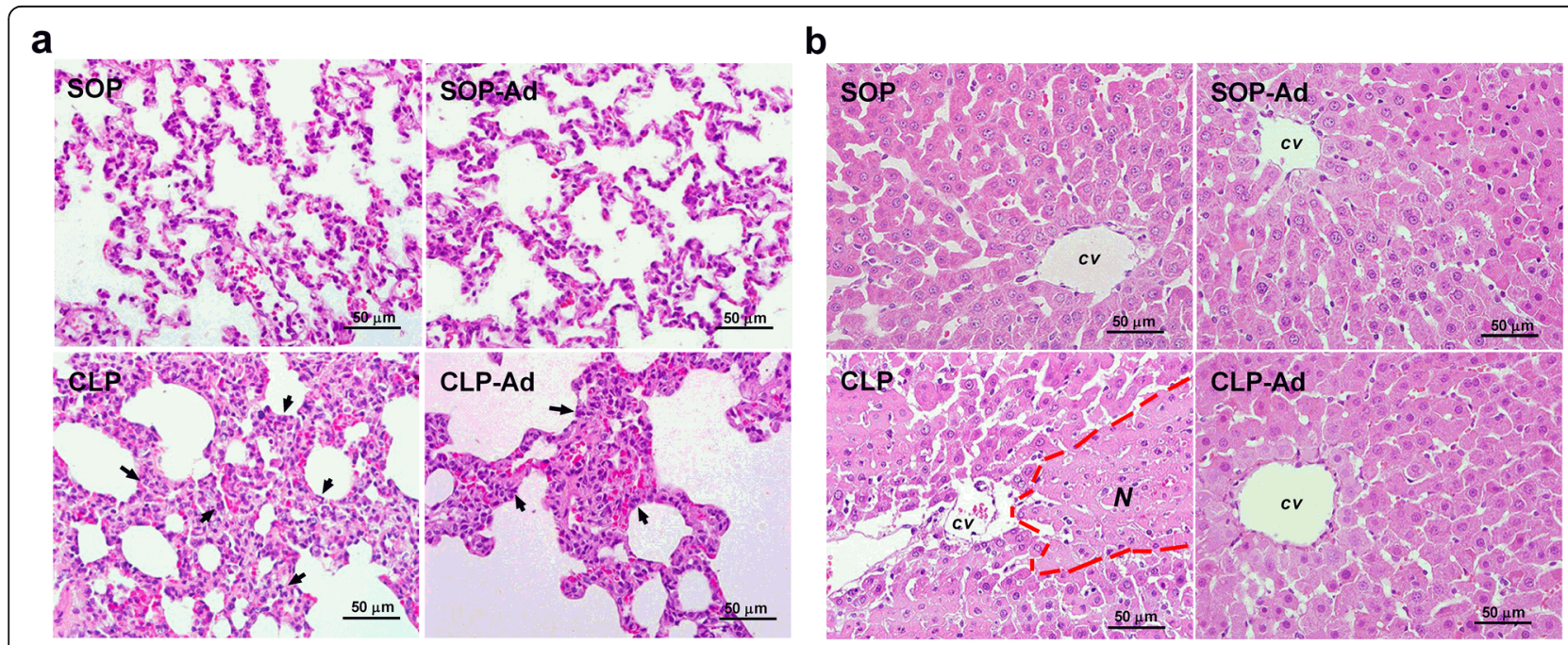

Fig. 6 Histopathological studies. Light microscopy of (a) lung and (b) liver from rats that underwent sham operation (SOP), SOP plus angiotensin(1-7) administration (1 mg/kg at 3 and $6 \mathrm{~h}$ after SOP procedure [SOP-Ad]), cecal ligation and puncture (CLP), and CLP plus angiotensin-(1-7) administration (CLP-Ad). Arrows indicate the infiltration of neutrophils. Hematoxylin and eosin staining showed significant necrosis in the liver at $24 \mathrm{~h}$ after the CLP procedure. Shown are representative micrographs from three rats per group. Magnification $\times 400 . N=$ Necrotic area, $C V=$ Central vein 


\section{Discussion}

This study demonstrated that the CLP procedure evoked delayed hypotension, hypoglycemia, and multiple organ injuries, characterized by elevated plasma biochemical parameters, histopathological changes, and mortality, which were improved by post-treatment with Ang-(1-7) in a rat model of peritonitis-induced sepsis. Further, Ang-(1-7) significantly attenuated plasma IL-6 production and lung superoxide production, as well as reduced liver IKB and caspase- 3 expression, in CLP rats. Thus, these findings suggest, for the first time, to our knowledge, that Ang-(1-7) reduces CLP-induced organ injury, inflammatory response, and even death and may be useful as a therapeutic agent targeting sepsis.

Ang-(1-7), mediated by the Mas receptor, has an anti-inflammatory effect on variable disease processes, such as atherosclerosis, arthritis, and asthma [4]. In an in vitro study, Ang-(1-7) reduced proinflammatory cytokine release in mouse peritoneal macrophages stimulated with LPS [14]. In an in vivo model of endotoxemia, LPS-induced hypothermia, consequent mortality [15], and muscle wasting [22] were attenuated or prevented by Ang-(1-7) through its Mas receptor. CLP is still considered to be the gold standard model of sepsis [23], although this model dose not reproduce completely the complexity of human sepsis. Our present study showed that CLP induced a significant increase of plasma IL-6 production, which was minimized by Ang-(1-7) administration. Moreover, ІкB protein, which inactivates the translocation of NF- $\mathrm{KB}$ into the nucleus, was enhanced by Ang-(1-7) in CLP rat liver. These results indicate that Ang-(1-7) prevents sepsis-induced inflammation through the IкB/ NF-kB pathway.

Increased activity of RAS could lead to oxidative stress and endothelial dysfunction in sepsis [24], which may be associated with the pathogenesis of pulmonary injury [9, 25, 26]. Concerning the counterregulatory actions on RAS, Ang-(1-7) attenuates the Ang II-stimulated increase in lipid peroxidation and decrease in superoxide dismutase activity in mouse heart [27]. In addition, pretreatment with Ang-(1-7) was found to diminish Ang II-induced ROS production in vascular smooth muscle cells [28]. Furthermore, our data showed that the CLP procedure significantly increased superoxide production in lungs, which was attenuated by the post-treatment of rats with Ang-(1-7). There was no significant difference observed in superoxide production in livers and kidney, and it is possible that the CLP procedure induced a trivial effect on these two organs by use of our method.

Recent studies demonstrate that increasing apoptosis in immune cells and parenchymal tissues appeared in the late stage of immunosuppression and consequent organ dysfunction in severe sepsis [16, 29]. Preventing cell apoptosis could improve survival in animal models of severe sepsis
[29]. Ang-(1-7) has been reported to inhibit Ang II- and LPS-induced apoptosis in endothelial and epithelial cells $[17,18,30,31]$. Our study found that Ang-(1-7) ameliorated caspase- 3 expression and necrosis changes in CLP rat liver. It indicates that Ang-(1-7) could attenuate apoptosis and cell death induced by severe sepsis. Additionally, apoptosis can be activated by diverse stimuli, including cytokines and ROS [32,33]. Therefore, Ang-(1-7) may exert its antiapoptotic effect by reducing the production of superoxide and inflammatory cytokines. Taken together, our results suggest that Ang-(1-7) attenuates subsequent organ dysfunction and improves survival in CLP-induced sepsis through its counterregulatory actions to mediate the resolution of inflammation, oxidative stress, and apoptosis.

A previous study showed that pretreatment of $1 \mathrm{mg} / \mathrm{kg}$ Ang-(1-7) inhibited LPS-induced cytokine production and hypothermia and thereby protected mice from the fatal consequences of endotoxemia [15]. In the present study, $1 \mathrm{mg} / \mathrm{kg}$ Ang-(1-7) was administered at $3 \mathrm{~h}$ after CLP procedure (Fig. 1), which did not attenuate organ dysfunction (Figs. 3 and 4). However, this dosage did not affect any hemodynamic and biochemistry parameters in sham control rats during the experimental period. Thereafter, we used post-treatment of $1 \mathrm{mg} / \mathrm{kg}$ Ang-(1-7) twice (i.e., at 3 and $6 \mathrm{~h}$ after CLP procedure) in the current study.

This study has several limitations. First, the CLP model was performed on young and healthy animals instead of in the clinical setting of elderly patients with comorbidities, although this model is considered the gold standard model of sepsis. Second, we did not use antibiotics in this study to avoid interfering with the effect of Ang-(1-7) on organ function. Third, Ang-(1-7) was administered starting $3 \mathrm{~h}$ after the CLP procedure, which does not represent clinical practice in which the therapeutic approach is usually undertaken in the late phase of sepsis. The effect of Ang-(1-7) used in the late phase of sepsis remains to be determined.

\section{Conclusions}

We describe a novel function of Ang-(1-7) in the therapy of CLP-induced sepsis. Ang-(1-7) attenuates multiple organ dysfunction and improves survival in rats with polymicrobial sepsis by reducing the inflammatory response, oxidative stress, and apoptosis characteristics, which may involve IкB/NF- $\mathrm{kB}$ signaling. Further studies, and particularly clinical trials, are necessary to clarify the potential adjunct effects of Ang-(1-7) in the early or even late phase of polymicrobial sepsis.

\section{Abbreviations}

ACE2: Angiotensin-converting enzyme 2; ALT: Alanine aminotransferase; Ang: Angiotensin; Ang II: Angiotensin II; BUN: Blood urea nitrogen; CLP: Cecal ligation and puncture; HR: Heart rate; IKB: Nuclear factor of kappa light polypeptide gene enhancer in B-cells inhibitor; IL-6: Interleukin 6;

LDH: Lactate dehydrogenase; LPS: Lipopolysaccharide; MAP: Mean arterial pressure; RAS: Renin-angiotensin system; SOP: Sham operation; TBST: Trisbuffered solution containing $0.1 \%$ Tween-20 


\section{Acknowledgements}

The authors thank Dr. Kuang-Yi Chang of the Department of Anesthesiology, Taipei Veterans General Hospital, and Ph.D., Division of Biostatistics, College of Public Health, National Taiwan University, for excellent assistance in performing the statistical analysis.

\section{Funding}

The project was funded by the Taipei Veterans General Hospital, Taiwan (V104C-046), and the Ministry of Science and Technology, Taiwan (MOST 105-2314-B-075-002-MY3).

\section{Availability of data and materials}

The datasets generated and/or analyzed during the current study are available from the corresponding author on reasonable request.

\section{Authors' contributions}

HJT and MHL analyzed the data and wrote the manuscript. MHL and CCS performed the in vivo experiments. SMK performed the histological examination. CMT conceived of the study, participated in the design of the study, interpreted the data, and revised the manuscript. CCW contributed to the design of the study and the writing of the manuscript. All authors read and approved the final manuscript.

\section{Ethics approval}

Approval of this study was received from Taipei Veterans General Hospital Animal Care Committee, Taiwan, ROC (IACUC 2015-003).

\section{Consent for publication}

Not applicable.

\section{Competing interests}

The authors declare that they have no competing interests.

\section{Publisher's Note}

Springer Nature remains neutral with regard to jurisdictional claims in published maps and institutional affiliations.

\section{Author details}

${ }^{1}$ Department of Anesthesiology, Taipei Veterans General Hospital and National Yang-Ming University, No. 201, Sec. 2, Shipai Road, Beitou District, Taipei 11217, Taiwan, Republic of China. ${ }^{2}$ Department of Pharmacology, National Defense Medical Center, Taipei, Taiwan, Republic of China. ${ }^{3}$ Graduate Institute of Aerospace and Undersea Medicine, National Defense Medical Center, Taipei, Taiwan, Republic of China. ${ }^{4}$ Department of Anesthesiology, Tri-Service General Hospital, National Defense Medical Center, Taipei, Taiwan, Republic of China.

Received: 9 May 2018 Accepted: 25 September 2018 Published online: 27 October 2018

\section{References}

1. Pro $\mathrm{Cl}$, Yealy DM, Kellum JA, Huang DT, Barnato AE, Weissfeld LA, et al. A randomized trial of protocol-based care for early septic shock. N Engl J Med. 2014:370:1683-93.

2. Zhou J, Qian C, Zhao M, Yu X, Kang Y, Ma X, et al. Epidemiology and outcome of severe sepsis and septic shock in intensive care units in mainland China. PLoS One. 2014;9:e107181.

3. Abraham E, Singer M. Mechanisms of sepsis-induced organ dysfunction. Crit Care Med. 2007;35:2408-16.

4. Passos-Silva DG, Verano-Braga T, Santos RA. Angiotensin-(1-7): beyond the cardio-renal actions. Clin Sci (Lond). 2013;124:443-56.

5. Santos RA, Ferreira AJ, Verano-Braga T. Angiotensin-converting enzyme 2, angiotensin-(1-7) and Mas: new players of the renin-angiotensin system. J Endocrinol. 2013;216:R1-17.

6. Santos RA, Simoes e Silva AC, Maric C, Silva DM, Machado RP, de Buhr I, et al. Angiotensin-(1-7) is an endogenous ligand for the $G$ protein-coupled receptor Mas. Proc Natl Acad Sci U S A. 2003;100: 8258-63.

7. Santos RA, Ferreira AJ, Pinheiro SV, Sampaio WO, Touyz R, Campagnole-Santos MJ. Angiotensin-(1-7) and its receptor as a potential targets for new cardiovascular drugs. Expert Opin Investig Drugs. 2005;14:1019-31.

8. Gallagher PE, Ferrario CM, Tallant EA. MAP kinase/phosphatase pathway mediates the regulation of ACE2 by angiotensin peptides. Am J Physiol Cell Physiol. 2008;295:C1169-74.

9. Imai Y, Kuba K, Rao S, Huan Y, Guo F, Guan B, et al. Angiotensin-converting enzyme 2 protects from severe acute lung failure. Nature. 2005;436:112-6.

10. Treml B, Neu N, Kleinsasser A, Gritsch C, Finsterwalder T, Geiger R, et al. Recombinant angiotensin-converting enzyme 2 improves pulmonary blood flow and oxygenation in lipopolysaccharide-induced lung injury in piglets. Crit Care Med. 2010;38:596-601.

11. Li Y, Cao Y, Zeng Z, Liang M, Xue Y, Xi C, et al. Angiotensin-converting enzyme 2 prevents lipopolysaccharide-induced rat acute lung injury via suppressing the ERK1/2 and NF-KB signaling pathways. Sci Rep. 2016;6:27911.

12. da Silveira KD, Coelho FM, Vieira AT, Sachs D, Barroso LC, Costa W, et al. Anti-inflammatory effects of the activation of the angiotensin-(1-7) receptor, MAS, in experimental models of arthritis. J Immunol. 2010;185:5569-76.

13. El-Hashim AZ, Renno WM, Raghupathy R, Abduo HT, Akhtar S, Benter IF. Angiotensin-(1-7) inhibits allergic inflammation, via the MAS1 receptor, through suppression of ERK1/2- and NF-KB-dependent pathways. Br J Pharmacol. 2012:166:1964-76.

14. Souza LL, Costa-Neto CM. Angiotensin-(1-7) decreases LPS-induced inflammatory response in macrophages. J Cell Physiol. 2012;227:2117-22.

15. Souza LL, Duchene J, Todiras M, Azevedo LC, Costa-Neto CM, Alenina N, et al. Receptor MAS protects mice against hypothermia and mortality induced by endotoxemia. Shock. 2014;41:331-6.

16. Aziz M, Jacob A, Wang P. Revisiting caspases in sepsis. Cell Death Dis. 2014; 5:e1526.

17. Ma X, Xu D, Ai Y, Zhao S, Zhang L, Ming G, et al. Angiotensin-(1-7)/Mas signaling inhibits lipopolysaccharide-induced ADAM17 shedding activity and apoptosis in alveolar epithelial cells. Pharmacology. 2016;97:63-71.

18. Li Y, Cao Y, Zeng Z, Liang M, Xue Y, Xi C, et al. Angiotensin-converting enzyme 2/angiotensin-(1-7)/Mas axis prevents lipopolysaccharide-induced apoptosis of pulmonary microvascular endothelial cells by inhibiting JNK NF-kB pathways. Sci Rep. 2015;5:8209.

19. Deitch EA. Animal models of sepsis and shock: a review and lessons learned. Shock. 1998;9:1-11.

20. Tsao CM, Chen SJ, Shih MC, Lue WM, Tsou MY, Chen A, et al. Effects of terbutaline on circulatory failure and organ dysfunction induced by peritonitis in rats. Intensive Care Med. 2010;36:1571-8.

21. Tsao CM, Li KY, Chen SJ, Ka SM, Liaw WJ, Huang HC, et al. Levosimendan attenuates multiple organ injury and improves survival in peritonitisinduced septic shock: studies in a rat model. Crit Care. 2014;18:652.

22. Morales MG, Olguin H, Di Capua G, Brandan E, Simon F, CabelloVerrugio C. Endotoxin-induced skeletal muscle wasting is prevented by angiotensin-(1-7) through a p38 MAPK-dependent mechanism. Clin Sci (Lond). 2015;129:461-76.

23. Dejager L, Pinheiro I, Dejonckheere E, Libert C. Cecal ligation and puncture: the gold standard model for polymicrobial sepsis? Trends Microbiol. 2011; 19:198-208.

24. Doerschug KC, Delsing AS, Schmidt GA, Ashare A. Renin-angiotensin system activation correlates with microvascular dysfunction in a prospective cohort study of clinical sepsis. Crit Care. 2010;14:R24.

25. Correa TD, Takala J, Jakob SM. Angiotensin II in septic shock. Crit Care. 2015; 19:98.

26. Klein N, Gembardt F, Supe S, Kaestle SM, Nickles H, Erfinanda L, et al. Angiotensin-(1-7) protects from experimental acute lung injury. Crit Care Med. 2013;41:e334-43.

27. Lin L, Liu X, Xu J, Weng L, Ren J, Ge J, et al. Mas receptor mediates cardioprotection of angiotensin-(1-7) against angiotensin I-induced cardiomyocyte autophagy and cardiac remodelling through inhibition of oxidative stress. J Cell Mol Med. 2016;20:48-57.

28. Zhang F, Ren X, Zhao M, Zhou B, Han Y. Angiotensin-(1-7) abrogates angiotensin Il-induced proliferation, migration and inflammation in VSMCs through inactivation of ROS-mediated PI3K/Akt and MAPK/ERK signaling pathways. Sci Rep. 2016:6:34621.

29. Luan YY, Yao YM, Xiao XZ, Sheng ZY. Insights into the apoptotic death of immune cells in sepsis. J Interf Cytokine Res. 2015;35:17-22.

30. Gopallawa I, Uhal BD. Angiotensin-(1-7)/mas inhibits apoptosis in alveolar epithelial cells through upregulation of MAP kinase phosphatase-2. Am J Physiol Lung Cell Mol Physiol. 2016;310:L240-8. 
31. Yang HY, Bian YF, Zhang HP, Gao F, Xiao CS, Liang B, et al. Angiotensin-(1-7) treatment ameliorates angiotensin II-induced apoptosis of human umbilical vein endothelial cells. Clin Exp Pharmacol Physiol. 2012;39:1004-10.

32. Elmore S. Apoptosis: a review of programmed cell death. Toxicol Pathol. 2007;35:495-516.

33. Wesche-Soldato DE, Swan RZ, Chung CS, Ayala A. The apoptotic pathway as a therapeutic target in sepsis. Curr Drug Targets. 2007;8:493-500.

Ready to submit your research? Choose BMC and benefit from:

- fast, convenient online submission

- thorough peer review by experienced researchers in your field

- rapid publication on acceptance

- support for research data, including large and complex data types

- gold Open Access which fosters wider collaboration and increased citations

- maximum visibility for your research: over $100 \mathrm{M}$ website views per year

At $\mathrm{BMC}$, research is always in progress.

Learn more biomedcentral.com/submissions 